Check for updates

London, UK

Cite this as: BMJ2020;370:m2717 http://dx.doi.org/10.1136/bmi.m2717 Published: 07 July 2020

\section{Covid-19: One in 10 cases in England occurred in frontline health and social care staff}

\author{
Ingrid Torjesen
}

Health and social care staff, hospital patients, and care home residents made up a substantial proportion of covid-19 infections in England during the height of the pandemic, a report by Data Evaluation and Learning for Viral Epidemics (DELVE) says.

The multidisciplinary group of researchers convened by the Royal Society estimates that around $10 \%$ of all covid-19 infections in England between 26 April and 7 June were among patient facing healthcare workers and resident facing social care workers (95\% confidence interval $4 \%$ to $15 \%$ ). ${ }^{1}$

"We estimate that care providers had around four times the risk of infection as similar working age people in that period," said Guy Harling, Sir Henry Dale senior research fellow at University College London and member of the DELVE working group, at a press briefing on 6 July. He emphasised that the figures only represented the six week period and the covid-19 situation was rapidly changing.

During that period, patients in hospital made up 11\% of all covid-19 cases, and residents in care homes a further $6 \%$. The estimates are based on population survey data from the Office for National Statistics combined with estimates of the population at risk, including workforce figures and data from the Vivaldi study. ${ }^{2}$

At least 1\% of all covid-19 infections in England were definitely (started at least 14 days after admission) or probably (started at least 7 days after admission) contracted by patients while in hospital, the researchers found. "This is a substantial proportion of the cases in hospital as a whole," Harling said.

Data from the Covid-19 Clinical Information Network show that an increasing proportion of cases were acquired in hospital between early March and early May, he added.

The report highlights a number of factors implicated in infections of staff, patients, and care home residents, including inconsistent use of masks and other personal protective equipment (PPE); lack of access to testing; lack of physical distancing between staff and patients, not just on wards but also in corridors, officers, and canteens; environmental and hygiene problems, including disinfection of surfaces and ventilation; difficulties in avoiding mixing infected and uninfected patients; rotation of staff between different locations; and inadequate surveillance systems both to investigate individual infections and wider outbreaks.

The researchers called for "an ambitious and comprehensive approach" similar to the one that was successfully implemented to combat meticillin resistant Staphylococcus aureus, but one that achieved results in months rather than years.

Little was known about covid-19 when the pandemic hit England, and hospitals had taken important steps throughout to control infections, acknowledged Nigel Field, chair of the DELVE working group and director of the Centre of Molecular Epidemiology and Translational Research at the Institute for Global Health, University College London.

"We recognise the challenges of updating systems and practices amid responding to a new viral pandemic that none of us had heard of less than a year ago. We recognised that there were problems with the supply of PPE and testing, particularly in the early phases of the pandemic, and although these problems have been largely resolved they will remain important for any future waves," he said.

The researchers said that health and social care workers were at heightened risk not just because of occupational exposure but also from other factors such as greater use of public transport and greater likelihood of living with another health or social care worker.

“We don't know what part was occupationally acquired or was acquired in transport or at home or elsewhere. This is challenging to understand, at an individual level and at a population level," Field told The BMJ. "We think an important fraction is probably acquired occupationally because healthcare workers are perhaps most likely to be exposed at work, but I don't think we have the data yet to draw firm conclusions about the fraction of occupationally acquired covid-19 in healthcare workers."

Anne Johnson, professor of infectious disease epidemiology at University College London, vice president of the Academy of Medical Sciences, and a member of the DELVE committee said, that the report was "deliberately timed" to help inform decisions around opening up of hospitals and other healthcare settings for non-covid-19 activities now that transmission had fallen and to prepare for winter and any future waves of covid-19

"This is a forward looking report with the idea that we must build resilience in relation to infections within health and social care systems and prevent infection in this essential sector in which a large proportion of the population are employed," she said. 


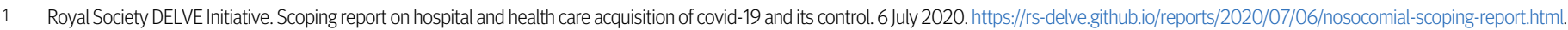

2 Shallcross L. Covid-19 in care homes (VIVALDI). ISRCTN Registry. 2020. www.isrctn.com/ISRCTN14447421.

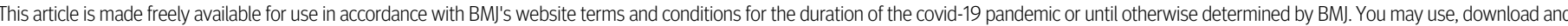
print the article for any lawful, non-commercial purpose (including text and data mining) provided that all copyright notices and trade marks are retained. 